AIAA-99-1933

\title{
FLIGHT TEST OF ASAC AIRCRAFT INTERIOR NOISE CONTROL SYSTEM
}

\author{
Dan Palumbo \\ Ran Cabell \\ John Cline \\ Brenda Sullivan
}

\author{
NASA Langley Research Center \\ National Research Council \\ U.S. Army Research Laboratory \\ NASA Langley Research Center
}

\begin{abstract}
A flight test is described in which an active structural/acoustic control system reduces turboprop induced interior noise on a Raytheon Aircraft Company 1900D airliner. Control inputs to 21 inertial force actuators were computed adaptively using a transform domain version of the multichannel filtered-X LMS algorithm to minimize the mean square response of 32 microphones. A combinatorial search algorithm was employed to optimize placement of the force actuators on the aircraft frame. Both single frequency and multi-frequency results are presented. Reductions of up to $15 \mathrm{~dB}$ were obtained at the blade passage frequency (BPF) during single frequency control tests. Simultaneous reductions of the BPF and next 2 harmonics of $10 \mathrm{~dB}, 2.5 \mathrm{~dB}$ and $3.0 \mathrm{~dB}$, were obtained in a multi-frequency test.
\end{abstract}

\section{$\underline{\text { Introduction }}$}

A flight test demonstrating interior noise reduction using active structural/acoustic control (ASAC) has been conducted on a Raytheon Aircraft Company Beech 1900D turboprop airliner. The ASAC approach has been in development for several years. $^{1,2,3,4,5}$ ASAC differs from the more common active noise control (ANC) ${ }^{6,7}$ approach in actuation method; ANC using loudspeakers versus ASAC's structural actuators. $^{8}$ For this flight test, inertial actuators were employed which were mounted directly to the 1900D frame. The ASAC approach has been pursued with the expectation that a mature design will be more cost effective than an ANC system of comparable performance. Efficiency improvements are expected in installation costs, channel count and channel power requirements.

Two new technologies were tested in the ASAC design. First, the filtered-x $\mathrm{LMS}^{9}$ controller was implemented in the principal component domain. ${ }^{10}$

\footnotetext{
Copyright (C) 1999 by American Institute of Aeronautics and Astronautics, Inc. No copyright is asserted in the United States under Title 17, U.S. Code. The U.S. Government has a royalty-free license to exercise all rights under the copyright claimed herein for Governmental purposes. All other rights are reserved for the copyright owner.
}

This provides processing efficiencies and controller stability beyond that of conventional controllers. Second, the actuator locations were optimized using combinatorial search techniques that were directed by predictions of noise reduction and control force. ${ }^{11}$ Proper positioning of ASAC actuators has been shown to be critical in achieving good noise control while using minimal force. ${ }^{11}$

The flight test objectives were to demonstrate stable noise control of the first 3 harmonics of the blade passage frequency (BPF) verifying controller performance and validating the optimization predictions. Both single frequency and multifrequency control were accomplished.

The following sections present a description of the principal component controller, the optimization procedure, and the test configuration. Results are presented and discussed, and concluding remarks offered.

\section{Principal Component Controller}

The principal component least mean squares (PCLMS) algorithm was used as the adaptive control algorithm for the flight test. This algorithm is a transform domain version of the multi-channel filtered-x LMS algorithm, ${ }^{9,12}$ and is described in detail elsewhere. ${ }^{10}$ In PC-LMS, the controller parameters (i.e. the filter weights) are adapted in a transformed coordinate system that decouples the multiple-input/multiple-output control channels at a single frequency. Each control channel in this transformed coordinate system is independent of every other channel. In contrast, the filter weights for the filtered-x algorithm are adapted in a coordinate system defined by the control actuators, which are not usually independent of one another and can show high degrees of inter-channel coupling when many actuators are used. By decoupling the control channels, convergence rates and control effort penalties can be set for each channel independently.

A block diagram of a feedforward controller based on the multiple error LMS algorithm is shown in Figure 1. The response of the error sensors is given by the 
(mx1) vector $\mathbf{e}$, and at a frequency $\omega$ as described by the expression

$$
\mathbf{e}(\omega)=\mathbf{H}(\omega) \mathbf{w}(\omega)+\mathbf{d}(\omega)
$$

The (rx1) vector $\mathbf{w}$ contains the control inputs to the actuators, and the (mxr) matrix $\mathbf{H}$ contains transfer functions from the input of each actuator to the output of each error sensor at the frequency $\omega$. The (mx1) vector $\mathbf{d}$ contains the error sensor responses to the primary noise field, and is called the primary response. The matrix $\hat{\mathbf{H}}(\mathrm{z})$ is an estimate of the physical error path transfer function matrix $\mathbf{H}(\mathrm{z})$, and is used to filter the reference signal. ${ }^{7}$

Each term in ( 1 ) depends on frequency $\omega$, and this dependence is understood implicitly in subsequent equations. The frequency domain representation in ( 1 ) describes the controller operating at steady state, with no transients, and should not be used to analyze the effect of delays in the error path transfer functions on the controller. ${ }^{12}$

The PC-LMS algorithm is obtained by substituting the singular value decomposition (SVD) of $\mathbf{H}$ into ( 1 ). The SVD of $\mathbf{H}$ is written

$$
\mathbf{H}=\mathbf{U S V}^{\mathrm{H}}
$$

where ()$^{\mathrm{H}}$ denotes the complex conjugate transpose. The (mxm) matrix $\mathbf{U}$ and (rxr) matrix $\mathbf{V}$ contain the eigenvectors of $\mathbf{H H}^{\mathrm{H}}$ and $\mathbf{H}^{\mathrm{H}} \mathbf{H}$, respectively. The (mxr) matrix $\mathbf{S}$ contains the square roots of the eigenvalues of $\mathbf{H}^{\mathrm{H}} \mathbf{H}$. The singular values are decreasing, such that $s_{1}>s_{2}>\ldots>s_{r}$, where $s_{i}$ is the ith singular value.

Substituting the SVD of $\mathbf{H}$ into ( 1 ) yields

$$
\begin{gathered}
\mathbf{e}=\mathbf{U S V}^{H} \mathbf{w}+\mathbf{d} \\
\mathbf{U}^{H} \mathbf{e}=\mathbf{S V}^{H} \mathbf{w}+\mathbf{U}^{H} \mathbf{d} \\
\zeta=\mathbf{S} \boldsymbol{v}+\mathbf{p}
\end{gathered}
$$

The vector $\boldsymbol{\zeta}=\mathbf{U}^{\mathrm{H}} \mathbf{e}$ represents the sensor responses mapped onto the principal components (PCs) of the control system, while $\mathbf{v}=\mathbf{V}^{\mathrm{H}} \mathbf{w}$ is the mapping of the actuator inputs onto the PCs. The vector $\mathbf{p}=\mathbf{U}^{\mathrm{H}} \mathbf{d}$ is the mapping of the primary field onto the PCs. The columns of the matrix $\mathbf{U}$ therefore constitute linear transformations from sensor responses to PCs, and the columns of $\mathbf{V}$ transform actuator inputs to PCs. ${ }^{10,12}$
Expanding ( 5 ) term by term produces,

$$
\zeta_{i}= \begin{cases}s_{i} v_{i}+p_{i} & \text { for } \mathrm{i}=1, \cdots, \mathrm{r} \\ p_{i} & \text { for } \mathrm{i}=\mathrm{r}+1, \cdots, \mathrm{m}\end{cases}
$$

assuming there are more error sensors than control actuators. Each PC error term, $\zeta_{i}$, depends only on the corresponding PC control input, $v_{i}$, and the mapping of the primary response onto the ith $\mathrm{PC}, p_{i}$. The last $(r+1)$ through $m$ PCs are not controllable, and constitute the residual field after control is applied.

Figure 2 contains a schematic of feedforward control implemented using the PC-LMS algorithm. The filter weights, $\mathbf{v}$, are adapted in terms of the PCs of the controller, and then transformed using $\mathbf{V}$ into actuator coordinates. The sensor responses are likewise transformed into PC coordinates using $\mathbf{U}^{\mathrm{H}}$ and used in the recursive updates of the filter weights in $\mathrm{PC}$ coordinates.

A recursive update for the $\mathrm{PC}$ control inputs, $v_{i}$, can be derived from the update expression used in the multiple error LMS algorithm. The resulting adaptive algorithm for the ith PC weight, $v_{i}$, is written ${ }^{10}$

$$
v_{i}(n+1)=v_{i}(n)-\mu_{i} s_{i} \zeta_{i}(n)
$$

Combining the scalar step size $\mu_{\mathrm{i}}$ and singular value $s_{i}$ into a single scalar yields a generalized update

$$
v_{i}(n+1)=v_{i}(n)-\alpha_{i} \zeta_{i}(n)
$$

where $\alpha_{i}$ is the step size parameter for the ith principal component.

It is often necessary to constrain the control outputs so they do not exceed physical limitations of the control actuators. There are two methods for constraining actuator inputs when the PC-LMS algorithm is used: (1) Set the step sizes, $\alpha_{i}$, of the last few PCs to zero. ${ }^{10}$ These PCs require the highest control effort. (2) Apply a control effort penalty or leak factor in the weight update recursion for some PCs. The update recursion with a control effort penalty is written

$$
v_{i}(n+1)=\left(1-\frac{u_{i}}{s_{i}} \beta_{i}\right) v_{i}(n)-\alpha_{i} \zeta_{i}(n)
$$

The noise reduction potential of candidate control systems is calculated during actuator location optimization. For a feedforward control system, predictions of noise reduction and control effort 
require knowledge of the transfer function matrix, $\mathbf{H}$, the primary response, $\mathbf{d}$, and an estimate of the coherence between the reference and the primary response, $\gamma^{2}$. The portion of the primary response at the ith microphone that is coherent with the reference signal, and therefore controllable, is written

$$
d_{i}^{c o h}=d_{i} \gamma_{i}
$$

where $\gamma_{i}$ is the coherence between the reference and the response of the ith microphone. Applying the PC transformation to the coherent portion of the primary response, $d_{i}^{c o h}$, produces a vector of coherent PC responses, denoted $p_{\mathrm{i}}^{\text {coh }}$. The predicted value of the control input to the ith $\mathrm{PC}$ is given by ${ }^{10}$

$$
v_{i}^{c o h}=\frac{-s_{i} p_{i}^{c o h}}{s_{i}^{2}+\beta_{i}}
$$

The predicted control inputs in terms of actuator coordinates can be computed from the PC control inputs, $\mathbf{v}^{\text {coh }}$, as

$$
\mathbf{w}=\mathbf{V} \boldsymbol{v}^{c o h}
$$

These control input values can be substituted into ( 1 ) to obtain the residual error. Noise reduction, in decibels, can then be computed by

$$
\Delta_{d B}=10 \log _{10}\left(\frac{\mathbf{e}^{H} \mathbf{e}}{\mathbf{d}^{H} \mathbf{d}}\right)
$$

\section{$\underline{\text { Test Configuration }}$}

The Raytheon 1990D, Figure 3, is one of the most widely used turboprop airliners in the industry. The aircraft can carry 19 passengers $2900 \mathrm{~km}$ at a maximum cruise speed of $533 \mathrm{kph}$. The 1900D has a 4 bladed propeller and associated blade passage frequency (BPF) of $\sim 103 \mathrm{~Hz}$. The twin engines are phase locked through a synchrophaser at the shaft speed of 25.8 revolutions per second (rps). The test aircraft was untrimmed.

A list of the flight test equipment is given in Table I and a block diagram of the system is shown in Figure 4. The control system used 32 microphones and 21 actuators. The controller, conditioners, amplifiers and digital tape recorder were arranged in 2 racks. The controller rack is shown in Figure 5.

\section{$\underline{\text { Controller }}$}

The controller consisted of a rack-mounted computer with digital signal processor (DSP), I/O and tachometer interface. The DSP board contained two TMS320C40 processors which were connected to three I/O boards. Each I/O board had 16 input and 8 output channels. A once-per-revolution pulse was obtained from the propeller shaft tachometer signal and used to trigger an interrupt on the DSP board. A phase lock loop tracked this interrupt signal and was used to generate a data sampling pulse at a frequency 48 times the once per revolution signal. With a nominal propeller shaft rate of $25.8 \mathrm{rps}$, the sampling rate for the control system was approximately 1238 Hz. Filters on-board the I/O boards were set to 723 $\mathrm{Hz}$ and provided $-18 \mathrm{~dB}$ roll-off per octave.

\section{Acquisition}

The primary response was sampled with $1 / 4$ " electret condenser microphones with $30 \mathrm{mV} / \mathrm{Pa}$ sensitivity. The microphones were uniformly distributed, 4 mics on a ring frame (as shown in Figure 6) with the lower and upper microphones roughly corresponding to seated and standing head heights respectively. In an effort to reduce near-field effects, the microphones were attached so that they protruded about $20 \mathrm{~cm}$ from the side wall. Eight frames in the passenger compartment of the aircraft were instrumented for a total of 32 microphones.

\section{$\underline{\text { Actuation }}$}

The inertial actuators were made especially for installation on an aircraft ring frame (see Figure 7). The actuators are designed to be mounted in pairs on the frame. Specifications for the actuator are summarized in Table III. Twenty-one actuator pairs were installed for the flight test.

The actuator resonant frequency ( $95 \mathrm{hz}$ ) was tuned to be just below the 1900D blade pass frequency (103 $\mathrm{Hz}$ ) to avoid the high rate of change of phase that typically occurs around resonance. The coil resistance $(7.5 \Omega)$ was chosen to be compatible with the multi-channel audio amplifiers that were used to power the actuators.

\section{Actuator Location Optimization}

The actuator locations were selected through a process of combinatorial search with a goal to reduce interior noise and control effort. The tabu search method, with its straightforward approach and 
success record, ${ }^{11}$ was chosen to optimize the actuator locations for the 1900D flight test.

A combinatorial search procedure such as tabu search combs through a large set of candidate actuator locations and selects a subset of a given size that best meets some goal or performance criteria. For the purpose of optimizing actuator locations for best noise reduction, a database of actuator acoustic response at each candidate location must be constructed and a procedure for predicting the noise reduction for any subset of actuator responses must be established.

The effectiveness of the tabu search algorithm hinges on the accuracy of the cost function (predicted noise reduction) and the coverage of the candidate set. An absolutely accurate cost function is not necessary for optimization. However, a good prediction is useful for making tradeoff studies of the number of actuators needed to meet a specific noise reduction goal. Acquiring the data necessary to construct the candidate set is challenging because it requires obtaining a representative sample of the actuator response at all the possible locations. A method of predicting the noise reduction which incorporates coherence and constraints is given in equations ( 11 ) through ( 13 ). The next section will describe how the actuator location candidate set was obtained.

\section{$\underline{\text { Actuator Location Survey }}$}

To optimize the actuator locations at a given frequency, a matrix of transfer functions between the input to an actuator at each candidate location and the microphone outputs must be assembled. Analytical methods are not yet capable of modeling the structural/acoustic response of an airframe with the fidelity needed to create this matrix, so an experimental approach was employed. This approach involved placing an actuator at each candidate location, driving it with a tone at the frequency of interest, and computing the transfer function to each microphone response. To avoid the time consuming process of drilling holes and bolting the actuator at each location, it was proposed that the actuator be clamped to the frame temporarily. The use of a single clamped actuator had the drawback that the relationship between the clamped actuator response and a bolted actuator-pair response was unknown. A preliminary test was performed which validated the use of the clamped actuator for the survey. The actuator/clamp assembly was able to be fitted to 82 locations on the 1900D frame. Thirty two microphones were mounted as described in the section on Test Configuration. Transfer functions were obtained at the BPF and 4 higher harmonics.

Pressurization Effects. The actuator location survey was done on the ground in an unpressurized cabin. The effects of cabin pressurization were of concern due to the associated changes in the structural/acoustic actuator transfer functions. If these changes were significant, they could invalidate the results of the actuator location optimization. Recent work $^{14}$ has demonstrated that pressurization changes can have a significant effect on the performance and stability of an ASAC system. Table IV shows noise control predictions for unpressurized and pressurized operating conditions. Both conditions use transfer functions obtained in unpressurized conditions. Based on these results, it can be expected that the actuator set optimized with ground-based transfer functions will not perform as predicted, especially in the higher harmonics. At the very least the transfer function data should be measured in flight, prior to the noise control experiment, to restore lost performance. However, selection of truly optimal actuator locations may require in-flight measurement of transfer functions for all candidate actuator locations.

Multi-Frequency Optimization. Multi-frequency actuator location optimization was accomplished using total noise reduction over the frequencies of interest as the search cost function. This cost function is written as

$$
J_{\text {tot }}=\sum_{i=1}^{n} a_{i}^{2} J_{i}
$$

where the parameters, $a_{\mathrm{i}}$, are defined according to the weighting method being used. Three weighting methods were evaluated: linear, dBA and loudness level. Loudness level weighting indicated there would be no subjective benefit from controlling the higher harmonics. Linear and A weighting showed similar effects, i.e., the $4^{\text {th }}$ and $5^{\text {th }}$ harmonic may not be worth controlling and very little benefit is gained from the $2^{\text {nd }}$ and $3^{\text {rd }}$. For these reasons the optimization was performed using the linear sum of the squared pressures of the first 3 harmonics to calculate the total noise reduction.

PC Optimization One of the features of the PC controller is that increased stability and performance can be achieved by not controlling the higher principal components which are associated with smaller singular values and higher control forces. This functionality can be simulated by using the PC 
domain noise prediction equations as given by Equations ( 10 ) through ( 13 ) as the optimization cost function.

As an example consider a 12 actuator, 32 microphone control system. Selecting a random set of actuators from the database of 82 possible locations results in values for the principal components of the primary field, $p_{i}$, as shown in Figure 8. This system will not achieve good noise reduction because too much acoustic power is concentrated in the higher order elements of $p_{i}$ (e.g. the $18^{\text {th }}$ ) which are either uncontrollable or difficult to control (due to small singular values). A set of actuators optimized using force constraints improves the design by shifting more acoustic power into the 12 controllable PCs, Figure 9. Here, the majority of the acoustic power is concentrated into the first few PCs, matching the authority available in the larger singular values.

\section{Optimization Results}

The actuator set used in the noise control flight tests were selected using a force constrained optimization (see section PC Optimization) over the first 3 harmonics. Figure 6 shows the numbering for some of the bays on the 1900D frame. Figure 10 shows all 21 actuator locations in a view where the frames are unwrapped, bay \#1 on the port or left side. The $1^{\text {st }}$ frame is closest to the cockpit, just behind the door, and is placed at the top of the figure.

The predicted noise reduction is listed in Table $\mathrm{V}$ for linear and A-weighted cases. Although the Aweighting produces a smaller overall noise reduction figure, the value of the $1^{\text {st }}$ harmonic reduction is identical to the linear case and the values of the $2^{\text {nd }}$ and $3^{\text {rd }}$ harmonics increase only slightly. This is further evidence of the dominance of the $1^{\text {st }}$ harmonic.

The noise controller used only the first 18 principal components due to processing limitations, so it was important to concentrate as much of the primary source in the first 18 principal components as possible. The principal component distribution of the first harmonic is well constructed (Figure 11). The $2^{\text {nd }}$ harmonic PC distribution is good (Figure 12), while the $3^{\text {rd }}$ harmonic PC distribution is only fair (Figure 13).

\section{$\underline{\text { Noise Control Results }}$}

The results of the noise control flight test are discussed here, including coherence data between the synchrophaser and the interior sound field, and noise reductions obtained at the first three harmonics of the propeller blade passage frequency. The section begins with a description of the test procedure used during the flight.

\section{Test Procedure}

The flight began with a climb to a cruising altitude of 15000 feet, at which point the pilot reduced the engine speed from the cruise engine speed of 1550 to $1440 \mathrm{rpm}$. This shifted the propeller blade passage frequency and its harmonics away from their cruise operating frequencies, thereby allowing the control system to measure the transfer functions between actuators and error sensors at the $1550 \mathrm{rpm}$ operating frequencies. The transfer functions were measured by exciting an individual actuator with one of the first three harmonics of the normal BPF while recording the microphone responses.

Once the system identification was completed, the pilot restored the engines to their cruise operating speed and the noise reduction tests were started. The test points obtained during the flight are listed in Table II. The first column in the table gives the run number for the test points; these numbers will be used subsequently when the results are discussed. The next two columns contain a description of each test point and the approximate length in minutes of each test. The notation BPF and 2BPF denote the blade passage frequency and its second harmonic, respectively. All of the test points listed in the table were conducted with the control system synchronized to the left (portside) engine. The total elapsed time from the start of the system identification procedure to touchdown was $79 \mathrm{~min}$.

\section{$\underline{\text { Coherence Results }}$}

The coherence between the reference signal and the primary noise field determines the maximum possible noise reduction in a feedforward control system. ${ }^{12}$ The reference signal was generated on the DSP synchronously with the tachometer signal taken from the port engine. One would therefore expect relatively good coherence between the reference and the noise field created by the portside propeller. Because the interior noise field contains contributions from the port and starboard propellers, the coherence may be reduced, dependent on the precision of the aircraft's synchrophaser. Another factor contributing to reduced coherence may be a lack of coherence between the engine tachometer signal and the interior noise field due to uncorrelated effects such as aerodynamic buffeting. 
Figure 14 shows the coherence between the reference and the microphone responses, as measured during test 5a (see Table II). The $\mathrm{x}$-axis denotes the microphone channel ranging from 1 to 32 , except for channel 7 which was inoperable during the tests and therefore is not plotted. The coherence is shown at the first three harmonics of the blade passage frequency, which are denoted as BPF, 2BPF, and 3BPF. The coherence values are generally high, and show a slight drop off with increasing harmonic number. One would expect to see greater microphone to microphone variation at the higher frequencies where the wavelengths are shorter.

\section{First Harmonic Results}

The first test concerned the reduction of the propeller blade passage frequency. As in all of the noise reduction tests, the control system was operated very conservatively; control effort constraints were set high initially, and were slowly reduced after the control system appeared to be stable. This resulted in long convergence times, which were acceptable for these tests since predictable, stable operation of the control system was more important than rapid convergence.

The uncontrolled sound pressure level (SPL) averaged across the microphone array is shown in Figure 15. The harmonics of the BPF are evident at 103, 206, 309, and $412 \mathrm{~Hz}$. The tone at $160 \mathrm{~Hz}$ was associated with the environmental control system on the aircraft and was not targeted by the noise reduction system during these tests. A time history of the average SPL of the blade passage frequency after control was turned on is shown in Figure 16. The solid line shows the measured SPL at approximately 1 second intervals during the 6.75 minute test. The other lines show the predicted noise reduction; the dashed line includes effort constraints and measured coherence, while the dotted line includes effort constraints but assumes unity coherence. The effort constraints were reduced incrementally during the test, hence the "stair-step" appearance of the predicted noise reduction.

The SPL of the BPF was reduced by nearly $15 \mathrm{~dB}$ six minutes after the control system was turned on. The measured reduction closely matches the dashed line, which illustrates the importance of coherence in noise reduction prediction.

Actuator Power. A primary concern in the design of the noise control system was to insure that the actuators had sufficient authority. Figure 17 is a plot of the percent of actuator maximum power consumed during this test for 10 of the most utilized actuators. At the end of the test a few actuators approached $60 \%$ power (about 7 watts), well below the 12 watt maximum. Using Figure 16 in conjunction with Figure 17, it is possible to tradeoff actuator power for noise reduction, thereby selecting a noise reduction figure that would require a less powerful, but cheaper actuator. Also notice that even though noise reduction is leveling off, that actuator power is climbing steadily, thus emphasizing the need for force constraints.

\section{Multi-Frequency Results}

Two multi-frequency tests were conducted in which the first three harmonics were controlled simultaneously. The goal of the first test (run $1 \mathrm{~b}$ in Table II) was to minimize a linear summation of the levels of the three harmonics, as given by ( 14 ). From the uncontrolled harmonic levels shown in Figure 15, this amounted to reducing the BPF first, then the second harmonic, and then applying any remaining control authority to the third harmonic. The goal of the second test (run 3a in Table II) was to minimize an A-weighted summation of the harmonic levels. This amounted to reducing the third harmonic, followed by the second, and then the BPF itself. The end result of these two tests was similar; small reductions were obtained at the two higher harmonics, because most of the control energy was spent where it was most effective, namely on the BPF.

Linear Cost Function. Time histories showing the reduction in a linear and an A-weighted summation of the three harmonics are plotted in Figure 18. The linear cost was reduced by slightly more than $8 \mathrm{~dB}(4$ $\mathrm{dBA}$ ) during the test. The test was approximately six minutes long, and produced noise reductions at the first three harmonics of $9.5 \mathrm{~dB}, 3.3 \mathrm{~dB}$, and $1.5 \mathrm{~dB}$, respectively.

A-weighted Cost Function. Measured reductions in the first three harmonics of $10 \mathrm{~dB}, 2.5 \mathrm{~dB}$, and $3.0 \mathrm{~dB}$, respectively, were obtained. Compared to the results from test $1 \mathrm{~b}$, there was an improvement at the third harmonic, from $1.5 \mathrm{~dB}$ to $3.0 \mathrm{~dB}$ of reduction, but this came at the expense of the second harmonic, which was reduced by only $2.5 \mathrm{~dB}$ in this test.

A time history of the cost function reduction is plotted in Figure 19. The A-weighted reduction of the three harmonics was just over $4 \mathrm{~dB}$, which is nearly identical to the reduction that was obtained in test $1 \mathrm{~b}$. The linear reductions were also nearly equal between the two tests. 
The actual multi-frequency noise reductions are listed in Table VI. The $1^{\text {st }}$ harmonic performs close to predictions (see Table $\mathrm{V}$ ), while the $2^{\text {nd }}$ and $3^{\text {rd }}$ harmonics performance falls well below. The similarity of this behavior to that of Table IV, indicates that cabin pressurization may have affected the optimization results.

\section{$\underline{\text { Concluding Remarks }}$}

The active structural/acoustic control system attained good control of the blade passage frequency in single frequency $(15 \mathrm{~dB})$ and multi-frequency $(10 \mathrm{~dB})$ tests. Control of the $2^{\text {nd }}$ and $3^{\text {rd }}$ harmonics did not meet expectations (expected $\sim 7$, obtained $\sim 3 \mathrm{~dB}$ ). This was due possibly to the effect of cabin pressurization on the structural/acoustic transfer functions. The transfer functions were obtained in unpressurized conditions on the ground and used for actuator location optimization. The resulting actuator array was probably sub-optimally placed, an effect having a greater impact at the higher harmonics. When inflight transfer functions are used, predictive capability is shown to be excellent. The use of the actuator array optimization technique can then be recommended if good transfer functions are available. The principal component control system proved to be highly configurable and remained stable throughout the test matrix. The ASAC approach has been shown to be very efficient requiring a maximum of 7 watts of power per actuator to produce $15 \mathrm{~dB}$ noise reduction in the $1^{\text {st }}$ harmonic.

\section{References}

1 C. R. Fuller and J. D. Jones. Experiments on reduction of propeller induced interior noise by active control of cylinder vibration. Journal of Sound and Vibration, 112:389-395, 1987.

2 Harold C. Lester and Sylvie Lefebvre. Piezoelectric actuator models for active sound and vibration control of cylinders. Journal of Intelligent Material Systems and Structures, 4:295-306, July 1993.

3 S. D. Snyder and C. H. Hansen. Mechanisms of active noise control by vibration sources. Journal of Sound and Vibration, 147(3):519525, 1991.

4 Richard J. Silcox, Sylvie Lefebvre, Vern L. Metcalf, Todd B. Beyer, and Chris R. Fuller.
Evaluation of piezoceramic actuators for control of aircraft interior noise. In DGLR/AIAA 14th Aeroacoustics Conference, Aachen, Germany, May 1992, number AIAA 92-02-091, pages 542-551, 1992.

5 D. R. Thomas, P. A. Nelson, and S. J. Elliott. Active control of the transmission of sound through a thin cylindrical shell, part II: The minimization of acoustic potential energy. Journal of Sound and Vibration, 167(1):113128, 1993.

6 S. J. Elliott, P. A. Nelson, I. M. Stothers, and C. C. Boucher. In-flight experiments on the active control of propeller-induced cabin noise. Journal of Sound and Vibration, 140(2):219238, 1990.

7 C.M. Dorling, G.P. Eatwell, S.M. Hutchins, C.F. Ross, and S.G.C. Sutcliffe., A Demonstration of Active Noise Reduction in an Aircraft Cabin. Journal of Sound and Vibration 128(2):358-360, 1989.

8 C.F. Ross and M.R.J. Purver, Active cabin Noise Control, Active 97, 39-46, Aug. 1997.

9 Stephen J. Elliott, Ian M. Stothers, and Philip A. Nelson. A multiple error lms algorithm and its application to the active control of sound and vibration. IEEE Transactions on Acoustics, Speech, and Signal Processing, ASSP35(10):1423-1434, October 1987.

10 Randolph H. Cabell. A Principal Component Algorithm for Feedforward Active Noise and Vibration Control. PhD thesis, Virginia Tech, Blacksburg, VA 24061, May 1998.

11 D. L. Palumbo and S. L. Padula. Optimization of an Actuator Array for the Control of MultiFrequency Noise in Aircraft Interiors. In $3 r d$ AIAA/CEAS Aeroacoustics Conference, Atlanta, May, 1997, number AIAA 97-1615, May 1997.

12 P. A. Nelson and S. J. Elliott. Active Control of Sound. Academic Press, 1992.

13 Dennis R. Morgan. An analysis of multiple correlation cancellation loops with a filter in the auxiliary path. IEEE Transactions on Acoustics, Speech, and Signal Processing, ASSP-28(4):454-467, 1980.

14 J.L. Caton, An Investigation Into the Control Robustness of Active Structural Acoustic Control in Aircraft Cabin Models. Masters Thesis, George Washington University. Aug. 1998 


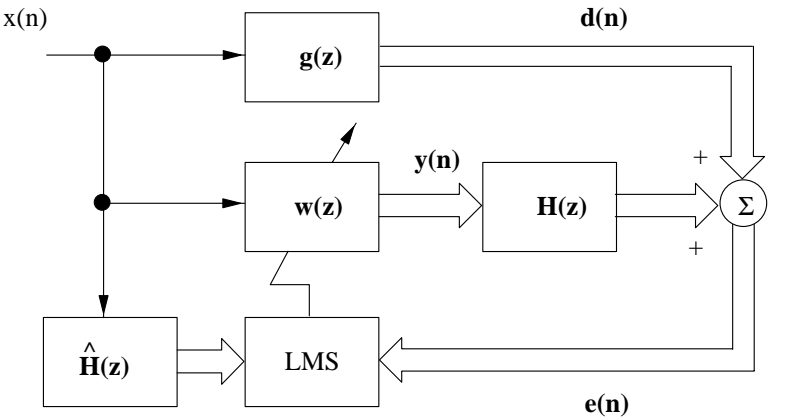

Figure 1. Multiple Error LMS Algorithm

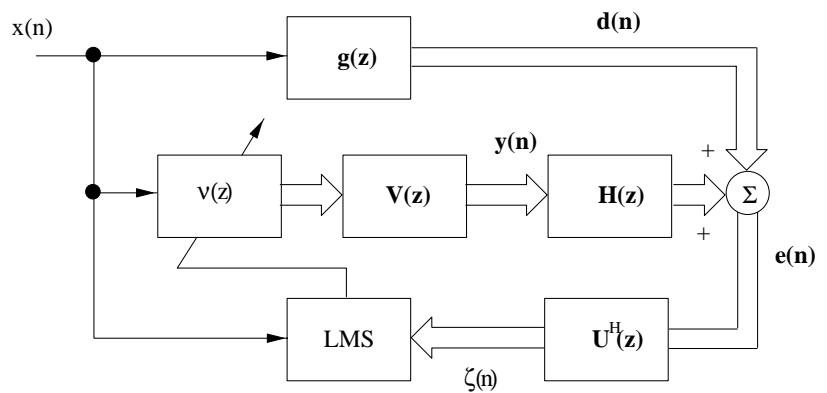

Figure 2. Principal Component LMS

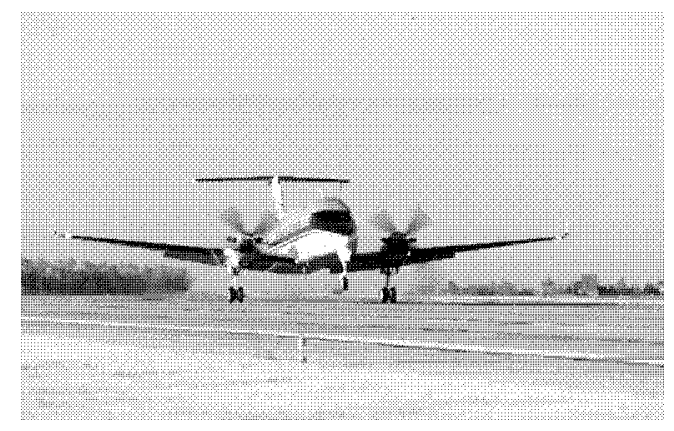

Figure 3. Raytheon/Beech 1900D

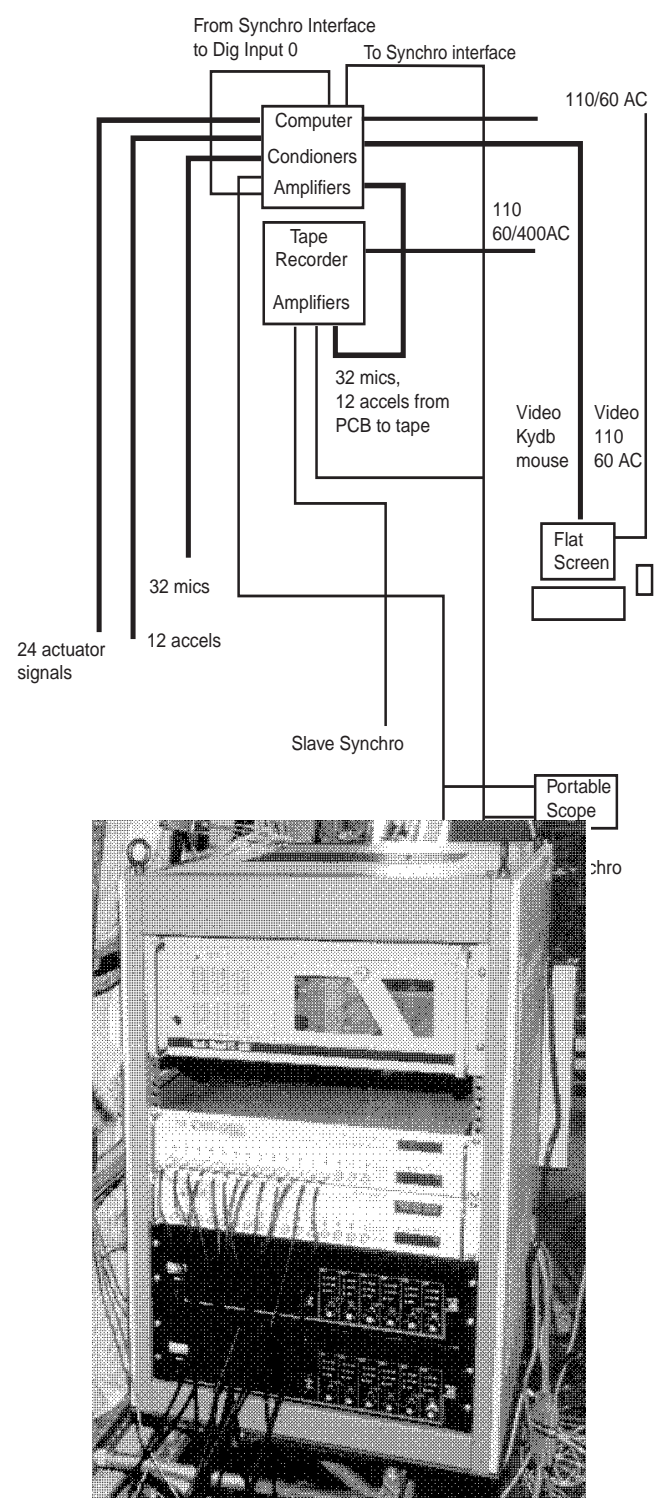

Figure 5. Controller Rack

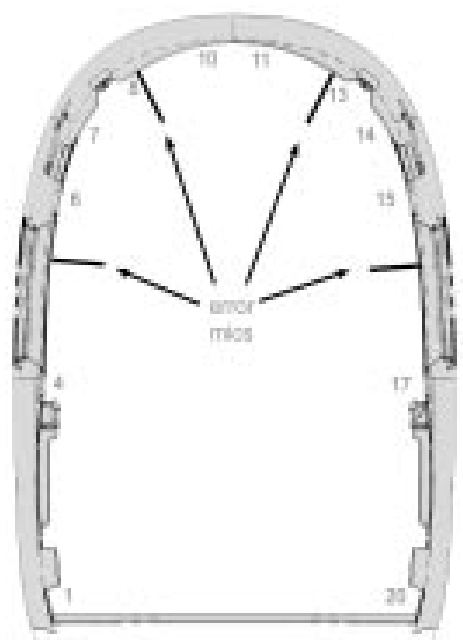

Figure 6. Frame bay and microphone locations

American Institute of Aeronautics and Astronautics 


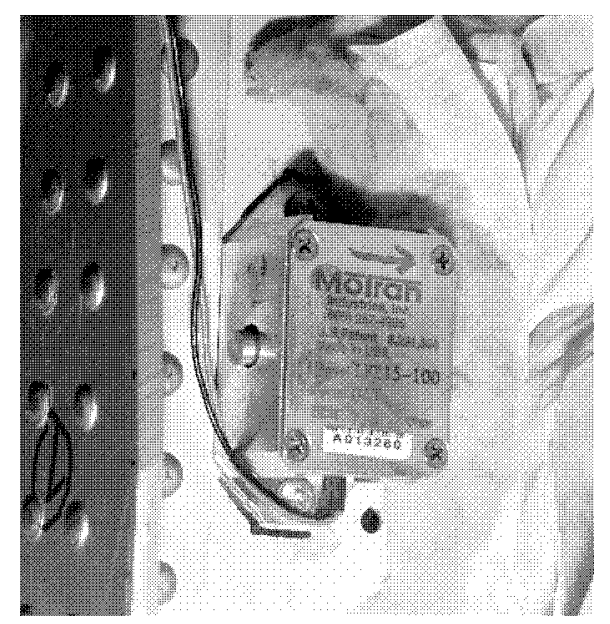

Figure 7. Inertial actuator mounted to $1900 \mathrm{D}$ frame.

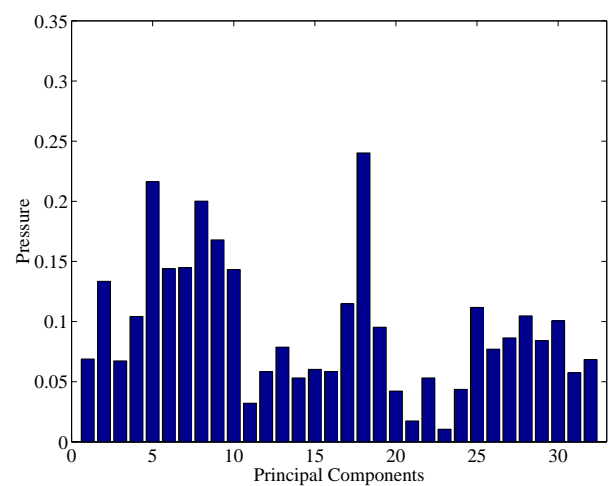

Figure 8. PCs using a random actuator set

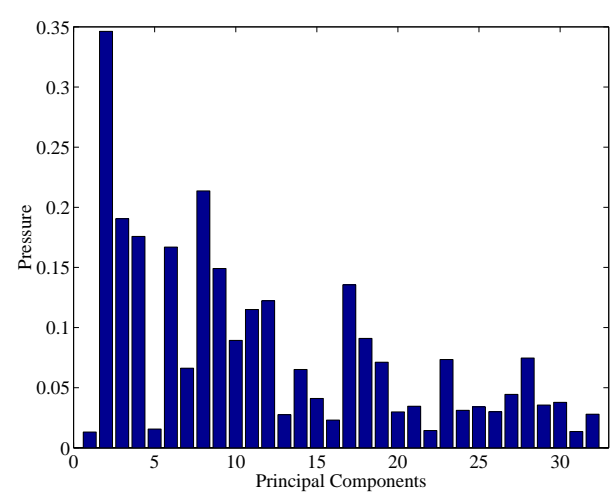

Figure 9. PCs using optimized actuators

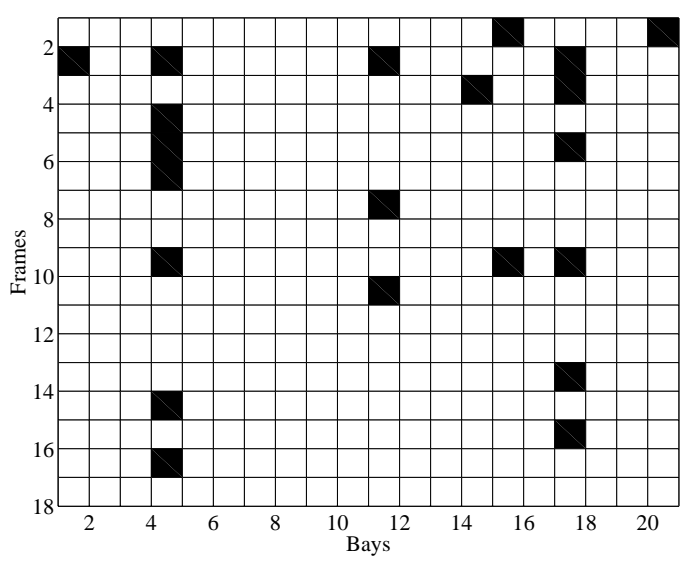

Figure 10. Actuator mounting locations on 1900D

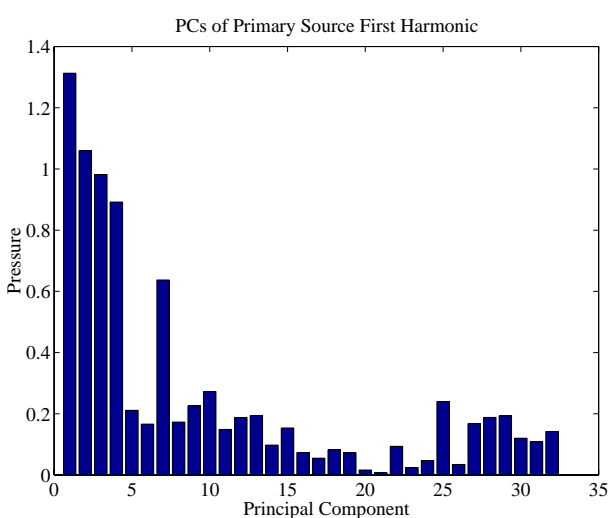

Figure 11. Primary PCs, $1^{\text {st }}$ harmonic

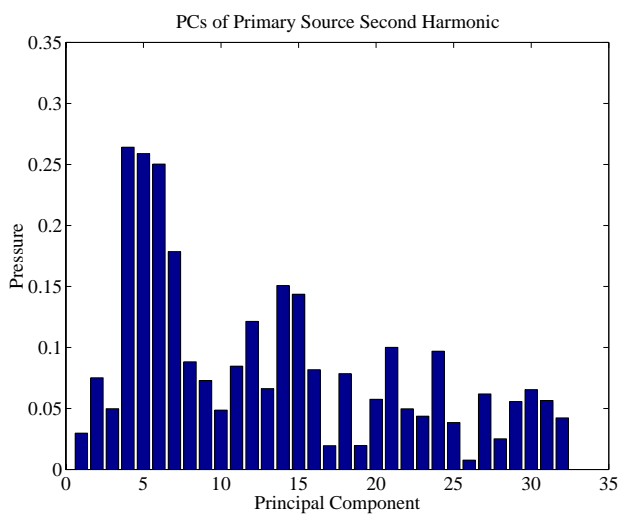

Figure 12. Primary PCs, $2^{\text {nd }}$ harmonic 


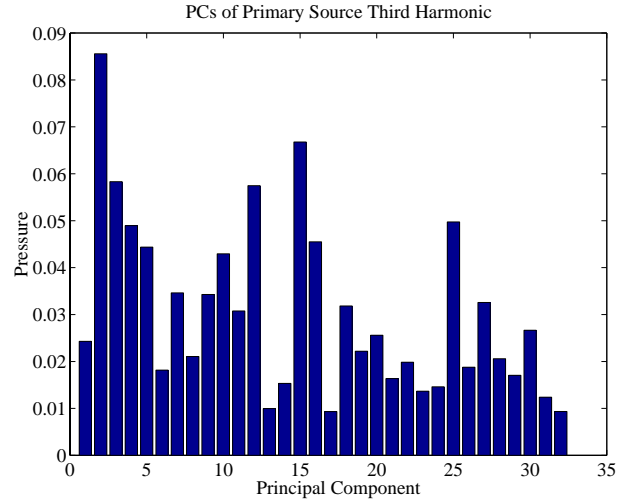

Figure 13. Primary PCs, $3^{\text {rd }}$ harmonic

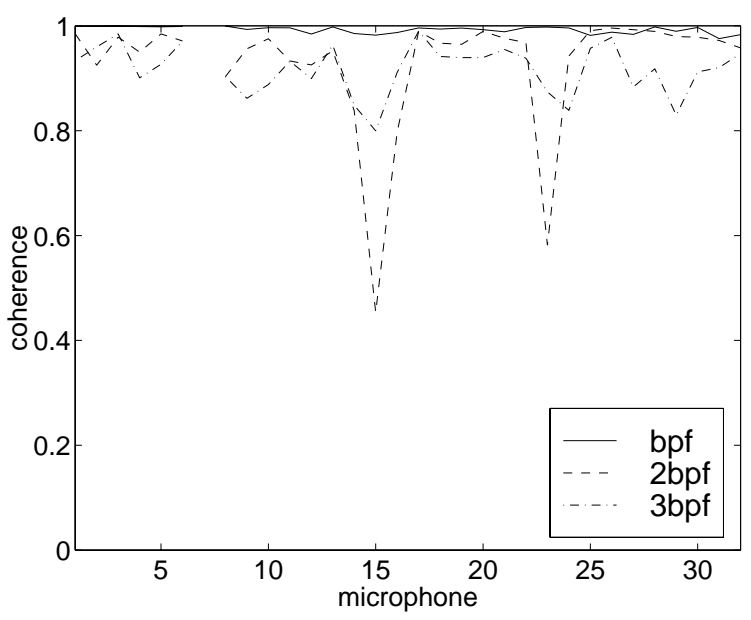

Figure 14. Reference to Primary Coherence, Run 5a

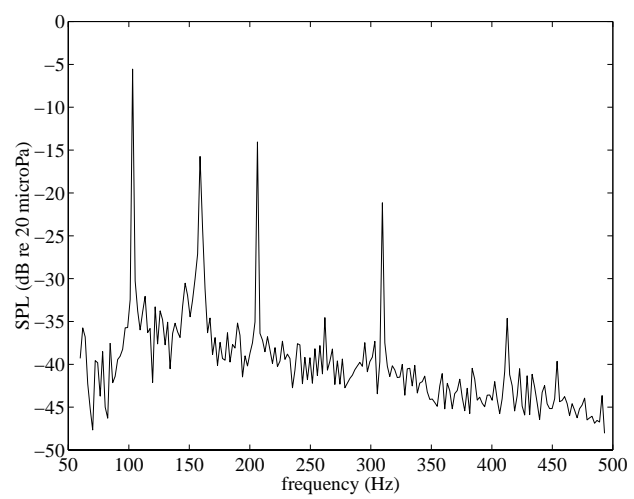

Figure 15. SPL befor control, run 1a

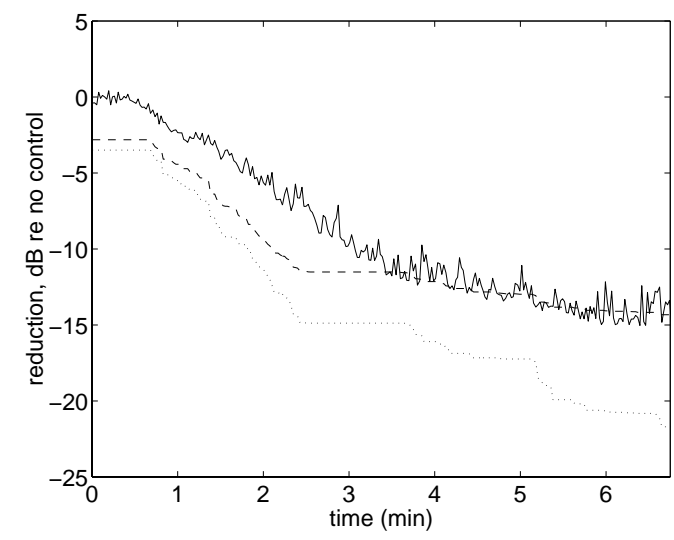

Figure 16. Reduction of BPF: -measured; --predicted; ... predicted using unity coherence

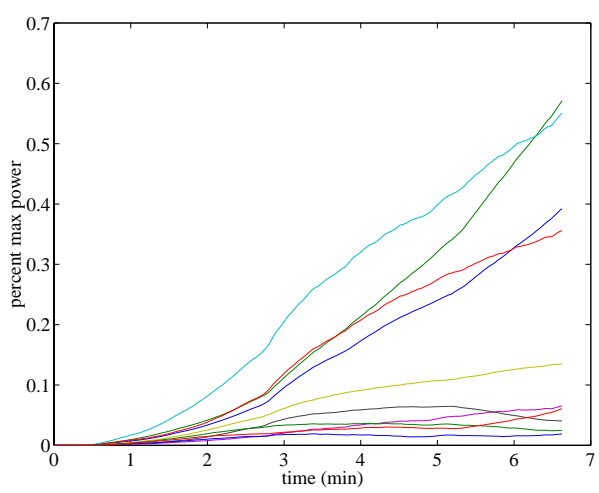

Figure 17. Actuator maximum power

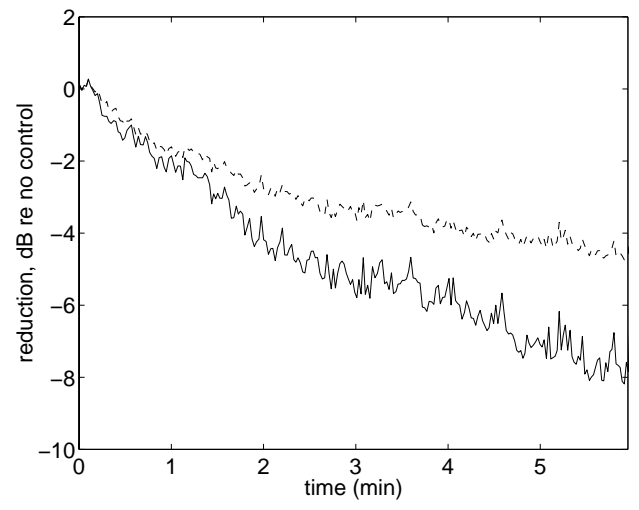

Figure 18. Reduction of cost function ( run $1 \mathrm{~b}$, linear weighted cost): -linear; --A-weighted 


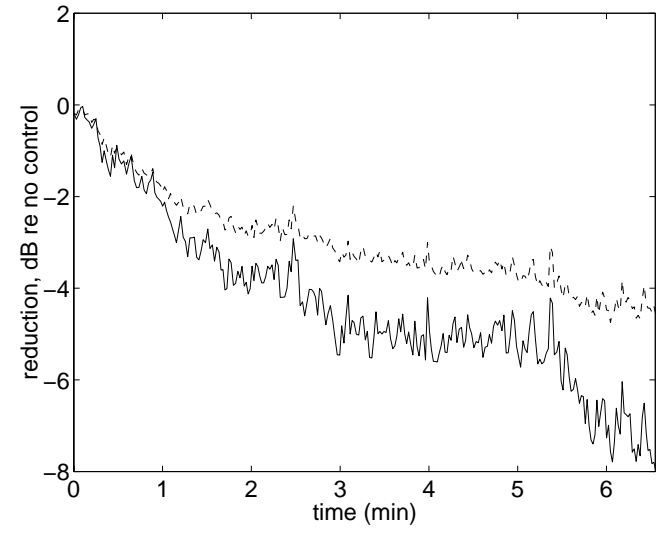

Figure 19. Reduction of cost function (run 3a, Aweighted cost): -linear; --A-weighted

\begin{tabular}{|c|c|c|}
\hline $\begin{array}{c}\text { Run } \\
\text { No. }\end{array}$ & Activity & $\begin{array}{c}\text { Time } \\
(\mathrm{min})\end{array}$ \\
\hline & System Identification & 28 \\
\hline 1a & control BPF & 7 \\
\hline 1b & control 1,2,3 BPF linear & 6 \\
\hline $3 \mathrm{a}$ & control 1,2,3 BPF A-weighted & 6 \\
\hline $5 \mathrm{a}$ & control 1,2,3 BPF linear, & 7 \\
& $8 \times 12 \mathrm{cfg}$ & \\
\hline $6 \mathrm{a}$ & control 2 BPF & 4 \\
\hline $7 \mathrm{a}$ & control 1,2,3 BPF, descent & 17 \\
\hline
\end{tabular}

Table II. Test Points

\begin{tabular}{|l|l|}
\hline Peak Force & $\begin{array}{l}75 \mathrm{~N}(17 \mathrm{lbf}) \\
@ 103 \mathrm{~Hz}\end{array}$ \\
\hline Power & $12 \mathrm{~W}$ \\
\hline Resistance & $7.5 \Omega(\mathrm{DC})$ \\
\hline Resonant Freq. & $95 \mathrm{~Hz}$ \\
\hline Weight & $245 \mathrm{gm}(0.5 \mathrm{lb})$ \\
\hline Dimensions & $64 \times 25 \times 36 \mathrm{~mm}$ \\
& $(2.5 \times 1 \times 1.4 \mathrm{in})$ \\
\hline
\end{tabular}

Table III. Specifications for IFX 15

\begin{tabular}{|c|c|}
\hline Item & Number \\
\hline \hline \multicolumn{2}{|c|}{ Controller } \\
\hline Rack-mount PC & 1 \\
\hline DSP Board & 1 \\
\hline I/O Board & $3 ; 48$ in; 24 out \\
\hline Tach Interface & 1 \\
\hline \multicolumn{2}{|c|}{ Acquisition } \\
\hline ICP Conditioners & $3 ; 48$ channels \\
\hline Microphones & 32 \\
\hline Accelerometers & 12 \\
\hline Tape Recorder & 48 channels \\
\hline \multicolumn{2}{|c|}{ Control } \\
\hline Amplifiers & $4 ; 24$ channels \\
\hline Actuators & 21 pairs \\
\hline \multicolumn{2}{|c|}{ Misc. } \\
\hline Oscilloscope & 1 \\
\hline Monitor & 1 \\
\hline Keyboard & 1 \\
\hline Mouse & 1 \\
\hline
\end{tabular}

Table I. Equipment List

\begin{tabular}{|l|c|c|c|c|c|}
\hline \multicolumn{1}{|c|}{ Condition } & $\begin{array}{c}104 \mathrm{~Hz} \\
\Delta \mathrm{dB}\end{array}$ & $\begin{array}{c}208 \mathrm{~Hz} \\
\Delta \mathrm{dB}\end{array}$ & $\begin{array}{c}312 \mathrm{~Hz} \\
\Delta \mathrm{dB}\end{array}$ & $\begin{array}{c}416 \mathrm{~Hz} \\
\Delta \mathrm{dB}\end{array}$ & $\begin{array}{c}520 \mathrm{~Hz} \\
\Delta \mathrm{dB}\end{array}$ \\
\hline \hline Unpressurized & -17.9 & -2.6 & -1.8 & -3.6 & -4.1 \\
\hline Pressurized & -13.1 & $\mathbf{6 . 1}$ & $\mathbf{1 . 1 3}$ & $\mathbf{9 . 8 6}$ & $\mathbf{5 . 2 3}$ \\
\hline
\end{tabular}

Table IV . Effect of cabin pressurization on predicted noise control

\begin{tabular}{|l|l|c|c|c|}
\hline Weighting & $\begin{array}{c}\text { Overall } \\
\Delta \mathrm{dB}\end{array}$ & $\begin{array}{c}\mathrm{BPF} \\
\Delta \mathrm{dB}\end{array}$ & $\begin{array}{c}2 \mathrm{BPF} \\
\Delta \mathrm{dB}\end{array}$ & $\begin{array}{c}3 \mathrm{BPF} \\
\Delta \mathrm{dB}\end{array}$ \\
\hline \hline Linear & -12.9 & -13.5 & -8.6 & -6.3 \\
\hline A-weighted & -10.8 & -13.5 & -8.7 & -6.4 \\
\hline
\end{tabular}

Table V. Predicted noise control for optimized actuator configuration

\begin{tabular}{|l|l|l|l|l|}
\hline Weighting & $\begin{array}{c}\text { Overall } \\
\Delta \mathrm{dB}\end{array}$ & $\begin{array}{c}\mathrm{BPF} \\
\Delta \mathrm{dB}\end{array}$ & $\begin{array}{c}2 \mathrm{BPF} \\
\Delta \mathrm{dB}\end{array}$ & $\begin{array}{c}3 \mathrm{BPF} \\
\Delta \mathrm{dB}\end{array}$ \\
\hline Linear & -8 & -9.5 & -3.3 & -1.5 \\
\hline A-weighted & -4 & -10.0 & -2.5 & -3.0 \\
\hline
\end{tabular}

Table VI. Actual multi-frequency noise control attained during runs $1 \mathrm{~b}$ (linear) and $3 \mathrm{a}$ (A-weighted) 